\title{
Magnitude entre a acessibilidade, espaço de tempo e o diagnóstico da tuberculose
}

Magnitude between accessibility, time period, and tuberculosis diagnosis Magnitud entre la accesibilidad, el espacio de tiempo y el diagnóstico de la tuberculosis

Angélica Aparecida Amarante Terra ${ }^{1}$ in https://orcid.org/0000-0001-7503-7877

Girlene Alves da Silva² ${ }^{2}$ ib htps://orcid.org/0000-0002-8758-1156

Márcio Roberto Silva ${ }^{3}$ i $n$ htps://orcid.org/0000-0002-0755-4415

Luciane Teixeira Passos Giarola ${ }^{4}$ (1) https://orid.org/0000-0002-8805-7583)

Isabel Cristina Adão Schiavon ${ }^{1}$ io https://orcid.org/0000-0003-1679-985x

Como citar:

Terra AA, Silva GA, Silva MR, Giarola LT, Schiavon IC. Magnitude entre a acessibilidade, espaço de tempo e 0 diagnóstico da tuberculose. Acta Paul Enferm. 2022;35:APE02692.

DOI

http://dx.doi.org/10.37689/acta-ape/2022A002692

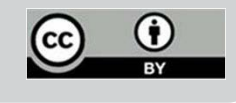

Descritores

Tuberculose; Acesso aos serviços de saúde; Tempo; Diagnóstico tardio; Avaliação de programas e projetos de saúde; Acesso aos serviços de saúde

Keywords

Tuberculosis; Health services accessibility; Time; Delayed diagnosis; Program evaluation; Health services accessibility

Descriptores

Tuberculosis; Accesibilidad a los servicios de salud; Tiempo; Diagnóstico tardio; Evaluación de programas y proyectos de salud; Accesibilidad a los servicios de salud

Submetido 21 de Setembro de 2020 Aceito

26 de Maio de 2021

Autor correspondente Angélica Aparecida Amarante Terra E-mail: angelica.terra@ifsudestemg.edu.br

Editor Associado (Avaliação pelos pares): Paula Hino (https://orcid.org/0000-0002-1408-196X) Escola Paulista de Enfermagem, Universidade Federal de São Paulo, São Paulo, SP, Brasil

\section{Resumo}

Objetivo: Analisar, na perspectiva dos portadores de tuberculose, a relação entre a acessibilidade ao sistema de saúde, o espaço de tempo e a realização do diagnóstico.

Métodos: Estudo analítico, correlacional, com delineamento transversal, realizado com 105 portadores de tuberculose atendidos na Atenção Primária à Saúde e no Serviço de Referência Especializado de um município prioritário mineiro. A análise de correspondência múltipla foi utilizada para identificar a associação entre os componentes da acessibilidade ao sistema de saúde, o espaço de tempo e a realização do diagnóstico da tuberculose.

Resultados: Observou-se associação temporal com o retardo no diagnóstico da tuberculose, com relação direta do serviço de primeira escolha do paciente, sendo os hospitais os locais que realizavam o diagnóstico em tempo oportuno, revelando baixa resolutividade dos serviços de atenção primária para as ações de controle da doença, no que se refere, principalmente, à identificação dos sintomáticos respiratórios.

Conclusão: Os achados destacam a urgência na reorganização dos serviços de atenção à tuberculose, fortalecendo a descentralização das ações para a Atenção Primária à Saúde, com enfoque na identificação precoce dos sintomáticos respiratórios e garantia de acessibilidade aos serviços de saúde, a fim de minimizar os impactos da extensão temporal no diagnóstico precoce.

\section{Abstract}

Objective: To analyze, from the perspective of tuberculosis patients, the relationship between accessibility to the health system, time period, and diagnosis.

Methods: This is an analytical, correlational study with a cross-sectional design was carried out with 105 tuberculosis patients treated in Primary Healthcare and in the Specialized Reference Service of a priority municipality in Minas Gerais. Multiple correspondence analysis was used to identify the association between the components of accessibility to the health system, time period, and tuberculosis diagnosis.

Results: A temporal association was observed with the delay in tuberculosis diagnosis, with a direct relationship between patients' first choice service, and hospitals were the places that gave diagnosis in a timely manner, revealing low resolution of primary care services for disease control actions, especially regarding the identification of respiratory symptoms.

Conclusion: The findings highlight the urgency in the reorganization of tuberculosis care services, strengthening the decentralization of actions for Primary Healthcare, focusing on the early identification of respiratory symptoms and ensuring accessibility to healthcare services, in order to minimize the impacts of temporal extension on early diagnosis.

'Instituto Federal de Educação, Ciência e Tecnologia do Sudeste de Minas Gerais, São João del-Rei, São João del-Rei, MG, Brasil.

2Universidade Federal de Juiz de Fora, Juiz de Fora, MG, Brasil.

${ }^{3}$ Empresa Brasileira de Pesquisa Agropecuária, Juiz de Fora, MG, Brasil.

4Universidade Federal de São João del-Rei, São João del-Rei, MG, Brasil.

Conflitos de interesse: nada a declarar. 


\section{Resumen}

Objetivo: Analizar, bajo la perspectiva de los portadores de tuberculosis, la relación entre la accesibilidad al sistema de salud, el espacio de tiempo y la realización del diagnóstico.

Métodos: Estudio analítico, correlacional, con diseño transversal, realizado con 105 portadores de tuberculosis atendidos en la Atención Primaria de Salud y en el Servicio de Referencia Especializado de un municipio prioritario del estado de Minas Gerais. El análisis de correspondencia múltiple fue utilizado para identificar la relación entre los componentes de la accesibilidad al sistema de salud, el espacio de tiempo y la realización del diagnóstico de tuberculosis.

Resultados: Se observó asociación temporal con el retraso del diagnóstico de la tuberculosis, con relación directa del servicio de primera opción del paciente, de los cuales los hospitales era el lugar que se realizaba el diagnóstico a su debido tiempo, lo que revela una baja resolución de problemas de los servicios de atención primaria para las acciones de control de la enfermedad, principalmente respecto a la identificación de los sintomáticos respiratorios.

Conclusión: Los resultados señalan la urgencia de reorganizar los servicios de atención de tuberculosis y fortalecer la descentralización de las acciones hacia la Atención Primaria de Salud, con enfoque en la identificación temprana de los sintomáticos respiratorios y garantía de accesibilidad a los servicios de salud a fin de minimizar los impactos de la extensión temporal del diagnóstico temprano.

\section{Introdução}

Ainda que os esforços estabelecidos pela Organização Mundial de Saúde (OMS) para o controle da tuberculose estejam sendo continuamente reestruturados, seus indicadores persistem elevados. ${ }^{(1)}$ No Brasil, em 2019, foram diagnosticados 73.864 casos novos da doença, o que corresponde à incidência de 35 casos $/ 100$ mil habitantes; e cerca de 2,2 óbitos/100 mil habitantes, o que correspondeu a 4.490 mortes em decorrência da tuberculose. ${ }^{(2)}$ Ao longo dos anos os coeficientes de incidência e mortalidade da tuberculose no Brasil tiveram um declínio, mas não foram suficientes para atingir e manter as metas estimadas pela OMS.

O controle eficaz da doença relaciona-se aos pacientes e serviços de saúde, na medida em que a dependência temporal influencia no sucesso para agilizar o diagnóstico. ${ }^{(3)}$ Esse tempo é determinante para a identificação precoce dos sintomáticos respiratórios e o início do tratamento no prazo adequado, evitando o agravamento do caso e aumentando as chances de cura. ${ }^{(4)}$

A magnitude temporal espelha a qualidade e resolutividade das estratégias de controle da tuberculose. ${ }^{(5)}$ A relação entre o primeiro contato do paciente com o serviço de saúde e sua acessibilidade, reflete diretamente na execução do diagnóstico. ${ }^{(6)} \mathrm{O}$ tempo é um dos fatores determinantes para avaliar a efetividade das açóes de controle da tuberculose, sendo influenciado por questóes relacionadas aos serviços de saúde, aos pacientes, ao perfil da comunidade e a situação epidemiológica da doença. ${ }^{(7)}$

Como umas das formas de possibilitar a acessibilidade aos serviços de saúde, a descentralização das medidas de controle para a Atenção Primária à
Saúde (APS) dinamiza algumas questóes referentes à doença, como identificação precoce dos sintomáticos respiratórios, proximidade dos serviços de saúde da residência dos pacientes, maior vínculo das equipes de saúde com a população, possibilitando demonstrar aspectos organizacionais e de desempenho que influenciam para a realização do diagnóstico em tempo oportuno. ${ }^{(6,8)}$

Diante do papel fundamental da realização do diagnóstico precoce da tuberculose para seguimento no itinerário terapêutico, o estudo busca responder em que aspectos a estrutura organizacional dos serviços de saúde assim como os elementos individuais relacionados ao doente retardam o diagnóstico da tuberculose? Nesse panorama, o objetivo desse estudo foi analisar, na perspectiva dos portadores de tuberculose, a relação entre a acessibilidade ao sistema de saúde, o espaço de tempo e a realização do diagnóstico da tuberculose.

\section{Métodos}

O presente estudo analisa e correlaciona, por um recorte transversal, o componente estrutural da acessibilidade aos serviços de atenção à tuberculose, sob a ótica de seus portadores. Realizado em um município prioritário para as açôes de controle da tuberculose do estado de Minas Gerais, que apresentou a segunda maior incidência da doença em 2018. ${ }^{(9)}$ Os dados foram coletados em unidades básicas de saúde e no Serviço Especializado em Tuberculose (Tisiologia), cenários acessíveis para a abordagem dos pacientes, uma vez que as consultas de controle mensais eram realizadas nestes locais. 
Foram convidados a participar do estudo, pacientes com diagnóstico para tuberculose, maiores de 18 anos e que iniciaram o tratamento há, pelo menos, um mês da data da coleta. Pelos critérios de seleção e o total da população elegível para o estudo, a amostra foi não probabilística do tipo voluntária, totalizando 105 participantes. Foram excluídos os portadores de tuberculose pertencentes ao sistema prisional; hospitalizados no período da coleta; portadores de transtornos mentais e aqueles que não foram encontrados durante o agendamento das visitas com, pelo menos, três tentativas de abordagem. Os participantes foram contatados por telefone ou através do Agente Comunitário de Saúde, sendo agendada a coleta dos dados e, nesta oportunidade, receberam explicaçóes sobre a pesquisa. Antes da coleta, assinaram o Termo de Consentimento Livre e Esclarecido (TCLE), em cumprimento à Resolução 466/2012.

A coleta ocorreu entre novembro de 2017 e dezembro de 2018 por uma equipe previamente capacitada. Foi utilizado o Primary Care Assessment Tool (PCATool) elaborado por Starfield, adaptado para atenção à tuberculose por Villa e Ruffino-Netto. $(10,11)$ Elegeu-se o "acesso ao diagnóstico" o componente avaliado neste estudo.

Foram utilizados os resultados de 6 variáveis, sendo elas: porta de entrada do sintomático respiratório no sistema de saúde, serviço de saúde que diagnosticou a doença, tempo para conseguir uma consulta, número de vezes que buscou o serviço para descobrir a tuberculose, tempo para confirmação do diagnóstico e busca pelo serviço mais próximo da residência. Conjuntamente realizou-se um levantamento socioeconômico, com questóes referentes à idade, sexo, escolaridade, renda, estado civil e situação de trabalho.

Para analisar a extensão temporal da realizaçáo do diagnóstico - sendo esta determinada desde a primeira busca pelo serviço de saúde até a obtenção do resultado laboratorial, e apresentar as correlaçóes entre as variáveis, foi criado indicadores que correspondem ao valor médio obtido pela somatória das respostas, sendo estas padronizadas para conseguir indicar o número de dias até a obtenção do diagnóstico, tendo como valor médio 23,5 dias $(\mathrm{DP} \pm 12,8)$.
Os dados foram tratados e analisados utilizando o software estatístico de livre domínio, o programa $\mathrm{R}$, sendo digitados por entrada dupla. Após a digitação, foi avaliada a consistência e edição do banco de dados, para eliminar a probabilidade de falhas na digitação.

Para estudar a associação e explorar a estrutura das relaçóes entre as variáveis, foi utilizada a Análise de Correspondência Múltipla, sendo construídos gráficos de correspondência. Nesses gráficos a associação entre categorias é observada através da localização de cada uma delas em relação aos eixos. De modo geral, categorias que se encontram no mesmo quadrante apresentam características comuns, indicando um perfil e os pontos localizados próximos à origem indicam associaçóes pequenas. Desse modo, quanto mais afastadas da origem e próximas umas das outras as categorias são, maior a associação entre elas. ${ }^{(12)}$

Esta pesquisa foi submetida e aprovada pelo Comitê de Ética em Pesquisa com parecer $\mathrm{n}^{\mathrm{o}}$ 2.085.06/2017, conforme a Resoluçáo no 466/12 (CAAE: 65955617.7.0000.5147).

\section{Resultados}

Participaram do estudo 105 portadores de tuberculose. $\mathrm{Na}$ análise do perfil socioeconômico, observa-se que a idade média dos participantes foi de 39 anos (DP $\pm 13,8)$, em sua maioria, homens $(55,2 \%$, $\mathrm{n}=58)$, adultos jovens $(56,1 \%, \mathrm{n}=59)$, não finalizaram o ensino fundamental $(29,6 \%, n=31)$, solteiros $(54,3 \%, \mathrm{n}=57)$, estavam desempregados $(41,9 \%$, $\mathrm{n}=44)$ e possuíam renda inferior a três salários mínimos $(69,6 \%, \mathrm{n}=73)$.

O gráfico de correspondência da figura 1 foi construído para avaliar a associação entre o serviço de saúde que diagnosticou a tuberculose, o tempo que o paciente demorou para conseguir uma consulta e o número de vezes que precisou procurar o serviço para descobrir a doença. Este gráfico explica $31 \%$ da variância dos dados.

Os pacientes diagnosticados em pronto atendimento estão associados a um tempo de espera de 5 dias ou mais para conseguir consulta e necessitam 


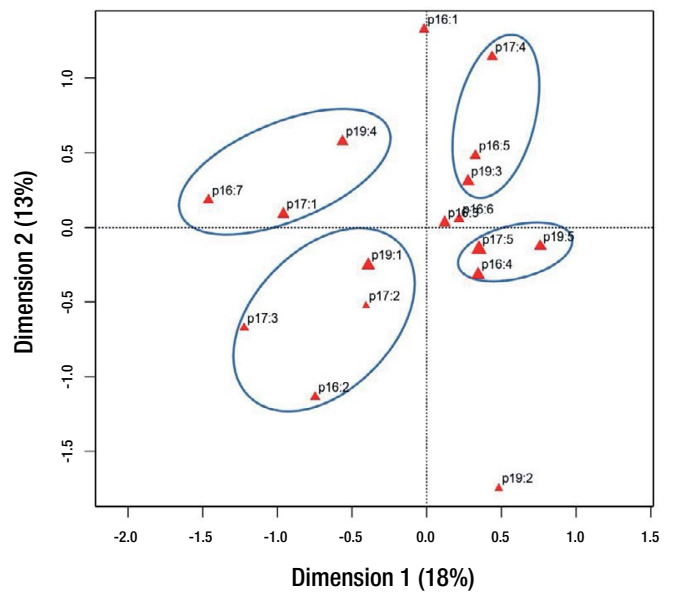

Legenda: Serviço de saúde que diagnosticou a TB $\rightarrow 16: 1$ - Estratégia Saúde da Familia (ESF); 16:2 - Unidades (Básicas de Saúde (UBS); 16:3 - Ambulatório de referência; 16:4 - Hospital público; 16:5 - Hospital privado; 16:6 Consultório particular; 16:7 - Pronto-atendimento; 16:8 - Outros. Tempo para conseguir uma consulta $\rightarrow$ 17:1 (5 dias ou mais); $17: 2$ - (4 dias); 17:3 - 3 dias); $17: 4$ - (2 dias); $17: 5$ - $(1$ dia - 24 horas). Número de vezes que buscou o serviço para descobrir a tuberculose $\rightarrow$ 19:1 - (5 ou mais vezes); 19:2 - (4 vezes); 19:3 - (3 vezes);

Figura 1. Associação entre o local que diagnosticou a tuberculose, 0 tempo para conseguir uma consulta e o número de vezes que o paciente buscou o serviço de saúde até receber 0 diagnóstico da tuberculose

procurar o serviço 2 vezes. Já aqueles diagnosticados em hospital público caracterizam-se por conseguir consulta em 1 dia e diagnosticarem a doença logo na primeira consulta. Nas Unidades Básicas de Saúde, os pacientes demoram de 3 a 4 dias para conseguir consulta e precisam procurar o serviço 5 vezes ou mais para serem diagnosticados. No hospital privado, os pacientes procuram o serviço de saúde 3 vezes para obter o diagnóstico e, em geral, demoram 2 dias para conseguir consulta.

Para avaliar a associação entre o primeiro serviço de saúde procurado pelo paciente quando apresentou sintomas de tuberculose e o local que diagnosticou a doença, utilizou-se o gráfico de correspondência da figura 2, o qual explica 33,2\% da variância dos dados.

Pode-se dizer que há uma associação entre procurar o hospital privado, quando os sintomas começam, e ser diagnosticado neste serviço de saúde, isto é, pacientes que procuram hospital privado com sintomas de tuberculose são diagnosticados principalmente neste hospital. A mesma associação pode ser observada para os consultórios particulares e hospital público. No caso do paciente buscar a Estratégia de Saúde da Família, o diagnóstico está principalmente associado à esta unidade de saúde, mas também ao pronto atendimento.

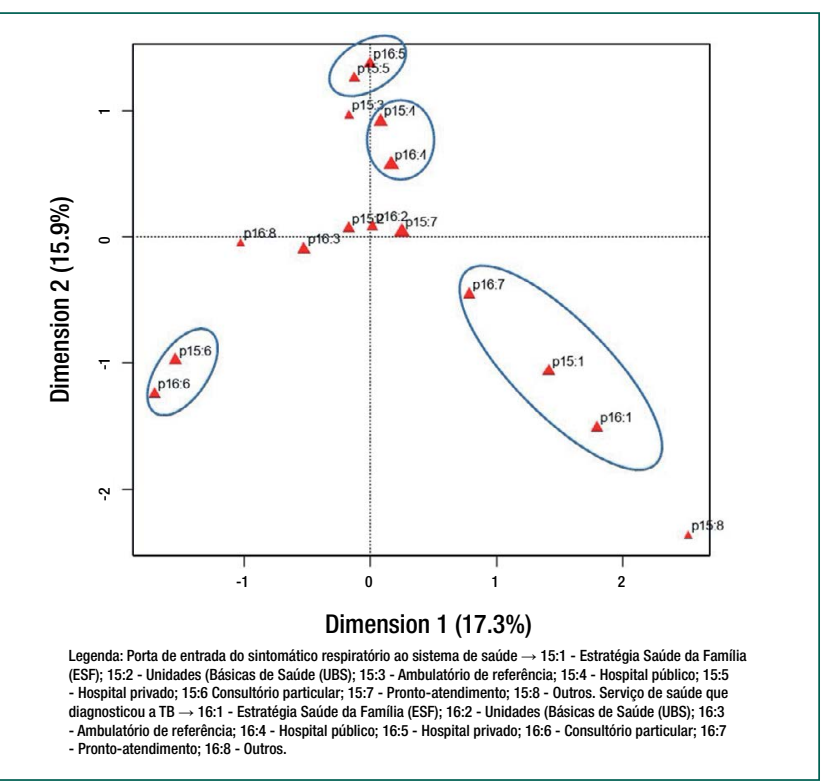

Figura 2. Acesso preferencial do sintomático respiratório ao sistema de saúde e o local que diagnosticou a tuberculose

Considerando o tempo que o paciente leva para descobrir a doença e o número de vezes que ele procura o serviço de saúde, podemos dizer, observando a figura 3, que os pacientes que procuram o serviço de saúde 5 ou mais vezes caracterizam-se por um tempo de 5 ou mais semanas para descobrir a doença. Já os que procuram a unidade de saúde apenas 1 vez estão associados a um tempo de 2 a 3 semanas para obter o diagnóstico.

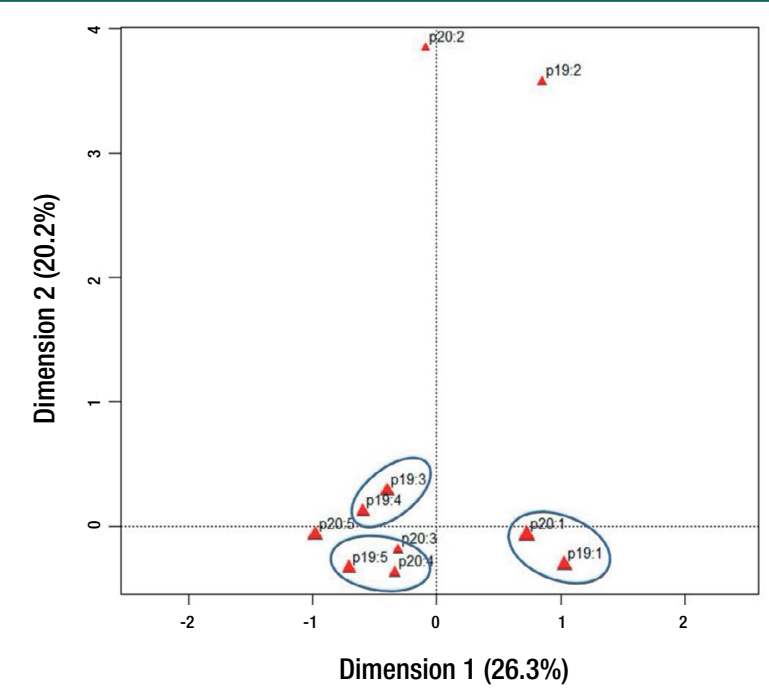

Caption: Número de vezes quebuscou o serviço para descobrir a tuberculose $\rightarrow$ 19:1 - (5 ou mais vezes); 19:2 - (4 vezes); 19:3 - (3 vezes); 19:4 - (2 vezes); 19:5 - (1 vez). Tempo para a confirmação do diagnóstico $\rightarrow$ 20:1 - (5 ou mais semanas); 20:2 - (4 semanas); 20:3 - (3 semanas); 20:4 - (2 semanas); 20:5 - (1 semana).

Figura 3. Relação temporal entre a busca pelo serviço de saúde e o diagnóstico da tuberculose 


\section{Discussão}

O estudo demonstrou que as açóes preconizadas para a garantia do itinerário terapêutico da tuberculose não são efetivas, iniciando pelo diagnóstico da doença. A acessibilidade aos serviços de saúde não é garantia de sucesso no controle da tuberculose, uma vez que a porta de entrada preferencial do paciente não ocorre pela APS, caracterizando baixa resolutividade, o que faz com o mesmo inicie sua saga em busca do atendimento assertivo por outros níveis de complexidade tecnológica, ampliando o tempo para a realização do diagnóstico.

As características socioeconômicas dos participantes reforçam o laço da doença com o fator social, reiterando, em sua maioria, indivíduos com pouca formação escolar, adultos jovens e desempregados, corroborando com pesquisas anteriores. ${ }^{(7)}$

O estudo aponta que na associação entre o local que diagnosticou a tuberculose e o tempo da resposta, foi possível perceber que os serviços de alta complexidade tecnológica, foram os que tiveram desempenho satisfatório na visão dos pacientes. Embora os programas de controle da tuberculose preconizem que o diagnóstico seja feito na APS, grande parte dos pacientes ainda são diagnosticados em hospitais. ${ }^{(6,13)}$ As barreiras de acessibilidade às unidades básicas de saúde e sua baixa resolutividade favorecem o aumento dos casos identificados em hospitais. ${ }^{(14)}$

Os obstáculos de acessibilidade concentram-se em aspectos da população e em características dos serviços de saúde. A busca pelo atendimento mais próximo da moradia e as condiçóes para deslocamento até as unidades de saúde constituem fatores que se relacionam aos usuários. A localização das unidades de saúde, as características estruturais dos serviços e a capacidade de atenção aos usuários são pontos que descrevem os aspectos institucionais da acessibilidade. $^{(6,7)}$ Em cada vertente, essas barreiras limitam o diagnóstico precoce da tuberculose, favorecendo o aumento da transmissibilidade e o agravamento do prognóstico do paciente.

Do ponto de vista da resolutividade, é perceptível que os serviços de atenção à tuberculose no município não satisfazem as demandas que surgem, tendo dificuldades para a implementação das estratégias que foram pactuadas. A resolutividade pode ser entendida como a avaliação satisfatória dos serviços obtidos pelo atendimento aos seus usuários. ${ }^{(15)}$ Essa análise envolve questóes relacionadas à organização e desempenho dos serviços de saúde, os níveis de complexidade tecnológica, a acessibilidade, a destreza dos profissionais, entre outros. ${ }^{(8)}$

As falhas nas açóes de controle da tuberculose na atenção básica contribuem para o crescente número de casos diagnosticados em hospitais que, muitas vezes, o paciente já apresenta quadro clínico avançado, com risco aumentado para a morte. ${ }^{(16)}$ Essa situação converge para o diagnóstico tardio da doença e a superlotação dos serviços de alta complexidade. ${ }^{(13)}$ A rede particular obteve destaque com a suspeição e rapidez no diagnóstico da tuberculose. Os pacientes que buscam esse serviço de saúde, o fazem pela lógica da resolutividade e agilidade com os casos, assim como, por terem uma condição socioeconômica mais favorável em relação aqueles que são diagnosticados nos ambulatórios de referência e nas unidades básicas de saúde. ${ }^{(17)}$

Observa-se com o estudo que os níveis de saúde com maior complexidade são capazes de identificar precocemente os casos, ao passo que aqueles pacientes que buscam a APS como primeira escolha para atendimento, o diagnóstico nem sempre ocorre neste local, estando também associado ao pronto-atendimento. Estudos evidenciam a relação entre o diagnóstico precoce e os serviços de saúde de primeiro contato, sendo as unidades de urgência e emergência as que os SR buscam com maior frequência quando do início do quadro clínico da tuberculose. ${ }^{(6,13,17)}$

Pela lógica da organização das redes de atenção à saúde, é característica da APS a porta de entrada do SR, constituindo-se eixo estruturante e ordenador do percurso da linha de cuidado. ${ }^{(8)}$ No entanto, essa premissa náo foi notória no presente estudo, no qual o SR busca, primeiramente, os níveis de maior complexidade tecnológica, configurando como alcance resolutivo para suas necessidades de saúde, como o caso das Unidades de Pronto Atendimento (UPA), que apresentam fluxo por demanda espontânea em 24 horas, dispondo de recursos de média 
complexidade tecnológica, que atendem às condições agudizadas de seus clientes. ${ }^{(6,13)}$

Considerando as sucessivas visitas pelo portador de tuberculose aos serviços de saúde para conseguir consultar e sua associaçáo com o tempo para a descoberta da doença, foi possível verificar que quanto mais o paciente busca os serviços de saúde para conseguir atendimento, maior é o tempo para a suspeição da tuberculose e a realização do diagnóstico. Sendo a tuberculose uma das doenças prioritárias no Brasil, as açóes de controle devem contar com estrutura e o desempenho mínimas das equipes e dos serviços de saúde, por meio do estímulo para a busca ativa dos casos e a percepçáo imediata dos SR, o acesso aos exames diagnóstico, o tratamento e as medidas preventivas. ${ }^{(5,6)}$

Os SR que buscam os serviços de saúde com sinais clássicos da tuberculose devem ser investigados, pois, muitas vezes esses sintomas são subestimados como problema de saúde pelos próprios pacientes, agravando o prognóstico e retardando a realização dos exames diagnósticos, que também apresentam barreiras no que tange ao tempo de retorno dos resultados. $^{(7,18)}$ A realização precoce do diagnóstico não deve exceder três semanas da identificação do $\mathrm{SR}$, iniciando o tratamento dos bacilíferos positivos em, no máximo, 48 horas após o resultado do exame. ${ }^{(19)}$ Essas condiçóes associam-se com os retornos sucessivos do paciente aos serviços de saúde para os devidos encaminhamentos do caso.

O atraso na realização do diagnóstico não está relacionado apenas às condiçóes do paciente. Sua efetivação pode estar relacionada ao tipo de serviço de saúde procurado pelo paciente como porta de entrada, que sugere a facilidade no acesso e a resolutividade do caso, com a percepção de atendimento imediato, recursos tecnológicos acessíveis e sensação de soluçáo do problema quando a procura ocorre preferencialmente pelos serviços de média e alta complexidade. ${ }^{(5,6,13)}$

A acessibilidade geográfica aos serviços de saúde influencia no tempo de realização do diagnóstico e condiciona às melhorias nos indicadores da tuberculose. ${ }^{(14,20)}$ A proximidade com a unidade de saúde satisfaz as necessidades do paciente, assegurando-lhe acolhimento e resolutividade, reforça-se seu vínculo com as equipes de saúde e, no controle da tuberculose, contribui para a identificação dos SR e para que os diagnósticos sejam realizados em tempo oportuno. ${ }^{(5,14)}$

Mesmo com os avanços na estruturação das políticas de controle da tuberculose, ainda perduram questôes para viabilizar a acessibilidade aos pacientes. ${ }^{(14)} \mathrm{E}$ necessário superar os desafios que contribuem para a ineficácia das açóes, como a baixa cobertura da APS no município do estudo (com cerca de 57,95\% da população total), a localização de algumas unidades de saúde em áreas de difícil acesso, a estrutura organizacional dos serviços de saúde, a limitação no horário de atendimento aos portadores de tuberculose, a infraestrutura inadequada para o acolhimento e atenção ao paciente, a incapacidade dos profissionais de saúde na suspeita clínica e na solicitação de exames para o diagnóstico precoce, entre outros. ${ }^{(9,14)}$

Faz-se necessário uma reorganização dos serviços de atenção à tuberculose, dando condiçóes para efetivação da descentralização para a APS, com o intuito de fortalecer sua capacidade organizacional para promoção da resolutividade de suas açóes, evitando o retorno constante do paciente aos serviços de saúde sem a realização do diagnóstico ou o seu deslocamento para os níveis de maior complexidade. Ainda, diante do cenário apresentado, sugere-se a ampliação da cobertura da APS; a capacitação contínua das equipes de saúde da atenção básica na suspeita clínica, priorizando as ações de prevenção na perspectiva de fortalecer a atenção básica para a ampliação da busca ativa dos casos e detecção precoce dos sintomáticos respiratórios, o que reduz o tempo para a realização do diagnóstico e o seguimento no itinerário terapêutico do doente.

Limitaçôes foram identificadas no estudo, sendo elas: demonstra a realidade de um município; algumas unidades de saúde têm barreiras geográficas que interferem na coleta de dados e, a dificuldade de encontrar os pacientes em sua residência.

\section{Conclusão}

O estudo apontou associação temporal com o retardo no diagnóstico da tuberculose, evidencian- 
do relação direta do serviço de primeira escolha do paciente, sendo os hospitais os locais que realizavam o diagnóstico em tempo oportuno. Essa realidade demonstra baixa resolutividade das açóes de controle da doença, especificamente na APS, impactando na realização precoce do diagnóstico e no prognóstico desfavorável do doente. É importante analisar os determinantes que impedem o fluxo contínuo da assistência, intensificando o acolhimento assertivo dos pacientes e as medidas organizacionais que estruturam os serviços de atendimento à tuberculose, para detecção precoce através da intensificação da busca ativa dos casos e suspeita clínica dos sintomáticos respiratórios, a fim de minimizar os impactos da extensão temporal para a realização do diagnóstico, promovendo a efetivação das medidas de controle da doença num intervalo de tempo aceitável para a continuidade do itinerário terapêutico.

\section{Colaborações}

Terra AAA, Silva GA, Silva MR, Giarola LTP e Schiavon ICA contribuíram com a concepção do estudo, análise e interpretação dos dados, redação do artigo, revisão crítica relevante do conteúdo intelectual e aprovação da versão final a ser publicada.

\section{Referências}

1. World Health Organization (WHO). Global tuberculosis report 2020. Genève: WHO; 2020 [cited 2020 Jan 20]. Available from: https://apps. who.int/iris/bitstream/handle/10665/336069/9789240013131-eng. pdf

2. Brasil. Ministério da Saúde. Secretaria de Vigilância em Saúde. Departamento de Doenças de Condições Crônicas e Infecções Sexualmente Transmissíveis. Boletim Epidemiológico de Tuberculose. Brasília (DF): Ministério da Saúde; 2020 [citado 2020 Jan 20]. Disponível em: http://www.aids.gov.br/pt-br/centrais-de-conteudos/ boletins-epidemiologicos-vertical

3. Macinko J, Almeida C, Oliveira E. Avaliação das características organizacionais dos serviços de atenção básica em Petrópolis: teste de uma metodologia. Saúde Debate. 2003;27(65):243-56.
4. Maciel EL, Golub JE, Peres RL, Hadad DJ, Fávero JL, Molino LP, et al. Delay in diagnosis of pulmonary tuberculosis at a primary health clinic in Vitoria, Brazil. Int J Tuberc Lung Dis. 2010;14(11):1403-10.

5. Pinheiro PG, Sá LD, Palha PF, Souza FB, Nogueira JA, Villa TC. Busca ativa de sintomáticos respiratórios e 0 diagnóstico tardio da tuberculose. Rev Rene. 2012;13(3):572-81.

6. Ponce MA, Wysocki AD, Scatolin BE, Andrade RL, Arakawa T, Ruffino Netto A, et al. Diagnóstico da tuberculose: desempenho do primeiro serviço de saúde procurado em São José do Rio Preto, São Paulo, Brasil. Cad Saúde Pública. 2013;29(5):945-54.

7. Trigueiro DR, Nogueira JA, Sá LD, Monroe AA, Anjos UU, Villa TC, et al. The influence of individual determinants in the delay of the tuberculosis diagnosis. Texto Contexto Enferm. 2014;23(4):1022-31.

8. Wysocki AD, Ponce MA, Brunello ME, Beraldo AA, Vendramini SH, Scatena LM, et al. Primary health care and tuberculosis: services evaluation. Rev Bras Epidemiol. 2017;20(1):161-75.

9. Rêgo RT, Assis AS, Costa RR. Perfil epidemiológico da tuberculose em Juiz de Fora, município prioritário no estado de Minas Gerais. HU Rev. 2018;44(3):343-50.

10. Starfield B. Atenção primária: equilíbrio entre necessidades de saúde, serviços e tecnologia. Brasília (DF): Unesco; Ministério da Saúde; 2002.

11. Villa TC, Ruffino-Netto A. Performance assessment questionnaire regarding TB control for use in primary health care clinics in Brazil. J Bras Pneumol. 2009;35(6):610-12.

12. Greenacre M, Blasius J. Multiple correspondence analysis and related methods. United Kingdom: Chapman \& Hall; 2006.

13. Paula R, Lefevre F, Lefevre AM, Galesi VM, Schoeps D. Why do tuberculosis patients look for urgency and emergency unities for diagnosis: a study on social representation. Rev Bras Epidmiol. 2014;600-14.

14. Clementino FS, Miranda FA. Acessibilidade: identificando barreiras na descentralização do controle da tuberculose nas unidades de saúde da família. Rev Enferm UERJ. 2010;18(4):584-90.

15. Turrini RN, Lebrão ML, Cesar CL. Resolutividade dos serviços de saúde por inquérito domiciliar: percepção do usuário. Cad Saúde Pública. 2008;24(3):663-74.

16. Ribeiro AS, Matsui TN. Hospitalização por tuberculose em hospital universitário. J Pneumol. 2003;29(1):9-14.

17. Dantas DN, Enders BC, Queiroz AA, Coura AS, Silva MP, Menezes RM. Factors related to the place of fi rst choice for the diagnosis of tuberculosis. Rev Gaúcha Enferm. 2014;35(3):75-81.

18. Scatena LM, Villa TC, Ruffino-Netto A, Kritski AL, Figueiredo TM, Vendramini SH, et al. Diffi culties in the accessibility to health services for tuberculosis diagnosis in Brazilian municipalities. Rev Saúde Pública. 2009;43(3):389-97.

19. Popolin MP, Rodrigues LB, Fronteira I, Yamamura M, Neto MS, Arcêncio R. Conhecimento sobre tuberculose, estigma social e a busca pelos cuidados em saúde. Rev Bras Pesq Saúde. 2015;17(3):123-32.

20. Maior ML, Guerra RL, Cailleaux-Cezar M, Golub JE, Conde MB. Time from symptom onset to the initiation of treatment of pulmonary tuberculosis in a city with a high incidence of the disease. J Bras Pneumol. 2012;38(2):202-9. 